\title{
Sous le signe de la qualité
}

Prof. Dr méd. Hans Ulrich Rothen, Dr méd. Roger Lussmann, Chantal Britschgi, Ursula Betschart

Membres de la Société Suisse de Médecine Intensive

En 2016, l'assemblée annuelle de la Société Suisse de Médecine Intensive (SSMI) s'est tenue du 21 au 23 septembre à Montreux. Comme en 2015, des experts de différentes disciplines et de nombreux intéressés ont participé à un séminaire d'une journée organisé pour les responsables de la gestion des services de soins intensifs. Les thèmes abordés n'étaient pas exclusivement centrés autour de la pratique de la médecine intensive, mais abordaient plus largement le fonctionnement du système de santé suisse dans son ensemble: le "ICU Management Seminar 2016» de la SSMI était résolument placé sous le signe de l'amélioration de la qualité.

\section{Quand des erreurs surviennent}

Une gestion efficace de la qualité commence par reconnaître le fait que même dans un système appliquant des standards très élevés, des erreurs peuvent survenir: "Même dans un système hautement complexe et technique comme la médecine intensive, un homme se trouve au centre, avec des capacités et ressources limitées», déclare Andreas Valentin, de la Société européenne de médecine intensive (ESICM). Par conséquent, garantir la sécurité des patients ne consiste pas seulement à prévenir les erreurs, une attitude parfois trop défensive qui peut elle-même générer des erreurs, mais également à savoir gérer les erreurs quand elles surviennent. Ainsi, la promotion d'une culture de l'erreur communicative et ouverte, qui inclut l'ensemble des employés (qu'ils soient soignants ou médecin-chef) et qui reconnaît les limites humaines et réduit la complexité de l'environnement, permet une amélioration efficace de la qualité.

\section{Remettre en question son propre travail grâce à des expertises externes}

Une évaluation critique par des personnes extérieures au service est également un instrument potentiellement utile pour améliorer la qualité de la prise en charge thérapeutique d'un service de soins intensifs. Dans ce type d'«évaluation par les pairs», un groupe d'experts externes, idéalement interprofessionnel, réalise une analyse du fonctionnement d'un service en tenant compte d'une multitude de critères. Pour Hans Ulrich Rothen, de l'hôpital universitaire de Berne, être un expert chevronné n'est pas essentiel pour cette analyse, et une préparation simple et la conduite de l'expertise comme proposé par l'Initiative pour une médecine de qualité (IQM) suffisent déjà pour contribuer à une amélioration de la qualité. Pour les experts eux-mêmes, cette expérience d'évaluation est très profitable, leur permettant de mieux connaître le travail et les processus d'un autre service et d'une autre clinique.

Tout comme Rosmarie Wyss, de l'hôpital universitaire de Berne, Anne-Claude Griesser du CHUV de Lausanne, où a eu lieu l'année dernière une première évaluation par les pairs menée par l'IQM, recommande également de telles expertises. Les évaluations par les pairs permettent la progression des compétences interprofessionnelles - l'une des grandes priorités de la SSMI-, car elles permettent aux médecins et aux soignants de collaborer encore plus étroitement. Les évaluations par les pairs sont également un bon moyen pour chacun d'évaluer la qualité de son travail et de définir des objectifs plus précis pour l'avenir.

A l'origine, le procédé d'évaluation par les pairs conçu par l'IQM à été développé pour le système de santé allemand. Avant l'introduction en Suisse, il était par conséquent nécessaire de réaliser quelques adaptations. Comme le souligne Pascal Besson de $\mathrm{H}+$, l'association des Hôpitaux de Suisse, un accent particulier a été mis pour garantir l'interprofessionnalité dans tout le processus, afin notamment de permettre à des soignants de s'investir en tant qu'experts.

\section{Promouvoir les talents pour affronter l'avenir}

La pratique d'une médecine intensive de qualité repose sur des collaborateurs hautement qualifiés. «La menace d'une pénurie de personnel a pour conséquence que la promotion des collaborateurs jouera un rôle encore plus 
important à l'avenir. Sur cet aspect, les hôpitaux peuvent encore apprendre quelque chose de l'économie», un point dont le professeur d'économie émérite Norbert Thom est convaincu. L'échec de la promotion de la relève dans les cliniques n'est souvent pas lié au manque de talent des plus jeunes, mais bien à l'attitude des supérieurs hiérarchiques. Leur manque d'engagement - fréquemment lié à une surcharge de travail - est dans bien de situations le point faible de la culture de promotion du talent au sein des cliniques. Des programmes de stage, tels qu'ils sont établis dans le secteur de l'économie depuis des décennies, peuvent également être mis en œuvre dans les hôpitaux afin d'attirer des collaborateurs à «haut potentiel», afin d'en faire non seulement des cadres dirigeants, mais également en conservant leurs qualités de professionnels de terrain, avec comme but idéal de les fidéliser. Norbert Thom ajoute: «Sans un réel engagement, même en haut lieu, par exemple au niveau du conseil d'administration, de tels programmes ne sont pas réalisables.»

«Les bons employés ne quittent pas des institutions, mais des personnes", affirme Walter Hagmann, de la Fachhochschule de Saint-Gall. Il existe certains facteurs de succès dans la promotion des employés particulièrement talentueux. Selon Walter Hagmann, la mise en place d'une culture de la confiance est absolument essentielle. Et laisser une certaine marge de manœuvre en fait également partie. Dans ce contexte, on parle volontiers d'empowerment (responsabilisation), ou la délégation d'une responsabilité, décisive pour que les collaborateurs à haut potentiel soient également en mesure d'évoluer par eux-mêmes et de se surpasser.

Dans l'assistance, des voix relèvent que la mise en œuvre d'un programme repris de l'économie pour la promotion des talents en médecine est difficile, car une clinique ne dispose que d'un nombre restreint de postes de cadres. Les talents doivent donc fréquemment chercher de nouvelles opportunités et fonctions à l'extérieur. Pour cette raison, il est souvent problématique en médecine de convaincre les personnes à haut potentiel de rester. Cependant, pour Christoph Haberthür, de la clinique Hirslanden à Zurich, c'est précisément la promotion des propres employés par les services d'autres cliniques qui doit être considérée comme un franc succès pour le service formateur, signant la qualité du travail et de la formation.

Le processus de promotion des talents dans l'environnement interprofessionnel de la médecine intensive ne doit pas oublier les soignants. Selon Mario Desmedt, de la Fondation Asile des Aveugles, il est particulièrement essentiel que le travail des soignants soit considéré comme important par les autres groupes professionnels, et que leur contribution soit appréciée et reconnue. D’après Irene Penker, de l'hôpital universitaire de Zurich, il faudrait en outre associer le personnel soignant aux décisions d'affectation des équipes; de cette manière, une institution peut devenir le point de départ d'un «hôpital-aimant» pour un personnel soignant hautement qualifié.

\section{Le DRG: un système avec un potentiel d'évolution}

Sans argent, pas de médecine intensive, et sans Diagnosis Related Groups (DRG), pas d'argent. Ces DRG ont certes pour objectif d'harmoniser le système de facturation au niveau national, mais tous les services de soins intensifs ne facturent pas leurs prestations de la même manière. Et c'est justement là que se situe l'un des problèmes du système DRG, selon Hervé Guillain, du CHUV de Lausanne. Dans la mesure où aucune unité de soins intensifs et aucun hôpital en Suisse n'est similaire, il apparaît difficile de développer une méthode de calcul des coûts qui s'applique à tous. Pour Simon Hölzer, de SwissDRG, le système a cependant un potentiel d'évolution. Et notamment, bien que les efforts d'harmonisation aient été importants, il subsiste une certaine hétérogénéité, notamment dans la collecte des données. «Bien entendu, cela complique quelque peu l'évolution du système», selon Simon Hölzer.

Jukka Takala, de l'hôpital universitaire de Berne, est persuadé que la saisie précise des prestations réalisées en médecine intensive est entravée essentiellement par le fait que $10 \%$ des patients longue durée mobilisent plus de 50\% des ressources, avec une comptabilisation trop peu précise par les DRG de l'investissement nécessaire à la prise en charge de ce petit groupe de patients critiques. C'est justement sur ce point que Simon Hölzer souhaite développer le dialogue entre SwissDRG et les professionnels, afin de permettre aux services de soins intensifs de Suisse de se comparer plus simplement, facilitant ainsi le benchmarking et permettant aux directions des cliniques de mieux montrer en quoi leur unité de soins intensifs contribue au succès global de l'hôpital, comme le déclare Jérôme Pugin, des Hôpitaux universitaires de Genève.

Pour Ursula Betschart, de l'hôpital cantonal de SaintGall, la manière la plus efficace d'assurer une sécurité financière est une qualité élevée du travail. Car «un client satisfait est un client qui revient». Conrad E. Müller, de la clinique Hirslanden à Zurich, conseille ainsi de ne pas trop se perdre dans les chiffres: «Nous ne devrions pas faire trop de mesures, mais plutôt simplifier les systèmes de mesure.» Sinon, il est possible de perdre de vue ceux qui devraient toujours se trouver au centre de notre attention: les patients en état critique et leurs proches. 